\title{
The potential of an automated system to identify the upper limb component of a controlled sitting posture
}

\section{Abstract}

Full trunk control in sitting is demonstrated only when the head-trunk are aligned and upper limbs remain free of contact from mechanical support. These components represent a Controlled Kinetic Chain and can be evaluated in people with neuromotor disability using the Segmental Assessment of Trunk Control (SATCo) when a therapist provides manual trunk support at different segmental levels. However, the SATCo, as with other clinical assessments of control, is subjective. The SATCo was translated to objective rules relating the position of the hands and elbows to the head-trunk and then tested to determine the extent to which this automated objective method replicated the clinical judgement.

Clinical evaluation used video to determine whether the upper limb was free of mechanical support while the objective evaluation used 3D motion capture of the trunk and upper limbs with a classification rule. The agreement between clinical and objective classification was calculated for three conditions of a distance-from-supportsurface threshold parameter in five healthy adults and five children with cerebral palsy.

The unfitted (zero-threshold values) method replicated the clinical judgement in part (68.26\% \pm 15.7 , adults, $48.3 \% \pm 33.9$ children). The fitted (level-of-support determined) agreement showed that the process could be refined using trial specific parameters (88.32\% \pm 5.3 adults, $89.84 \% \pm 10.2$ children). The fixed-values agreement showed high values when using general group parameters $(80.80 \% \pm 3.1$ adults, $74.31 \% \pm 21.5$ children).

This objective classification of the upper limb component of trunk control largely captures the clinical evaluation. It provides the first stages in development of a clinically-friendly fully automated method.

Key words

Trunk control; Clinical assessment; Automated method; Cerebral palsy; Controlled Kinetic Chain. 


\section{Introduction}

Independent unsupported sitting, with a vertically aligned head and trunk (head-trunk) is a milestone of typical development and requires full motor control of the head-trunk [1]. Reduction or absence of head-trunk control can result from neuromotor disability such as cerebral palsy (CP) with the consequent lack of independent sitting ability leading to functional limitations [1].

The head-trunk is a kinetic chain of segments comprising the head and neck and successive trunk segments to the pelvis. These axial segments branch into the upper limbs. The term 'Controlled Kinetic Chain' (CKC) denotes the biomechanical chain as a controlled entity and is used in the context of determining the neuromuscular control status of individual joints within that chain [2]. In independent unsupported sitting, full motor control of the whole kinetic chain of the head-trunk and upper limbs is demonstrated only when there is no end of range mechanical support at any axial joints or from external objects other than the primary support surface. This control without mechanical support is termed an Open-CKC [2]. In the trunk, a sitting posture that is, for example, slumped into full lumbar flexion with passive end of range mechanical support from intervertebral ligaments obviates the need for active control; it is termed a Closed-CKC [2]. This closure is assessed clinically by analysis of trunk alignment [3]. Use of the upper limbs or an external object to support the trunk mechanically can also remove the need for active control and is also termed a ClosedCKC [2]. This closure is assessed clinically by observation of the upper limbs in relation to the trunk and external objects. For example, if a person rests one hand on his/her thigh, then this can help maintain a sitting posture in the presence of poor trunk control even if the trunk is apparently aligned.

Assessment of trunk control should thus consider both alignment of the head-trunk segments and use of the upper limbs. In neuromotor disability such as $\mathrm{CP}$, motor control is usually assessed through comparison with typically developing children and inferring control status from functional activities $[4,5]$ or through a child's ability to maintain a balanced posture either statically and/or dynamically [6, 7]. The Segmental Assessment of Trunk Control (SATCo), uniquely assesses CKC status at six trunk segmental levels and free sitting [3]. Although it provides greater information about motor control strategies, in common with other clinical tests, it is subjective. Objective 
quantification is desirable since it is repeatable, eliminates variability between and within assessors and offers the potential for quantifying clinical changes over time. In order to complement a clinical assessment, an objective automated system should incorporate the rules existing in the specific clinical test. It should also be practical for clinical use and thus 'clinically-friendly' for both for the child and the therapist.

A method for quantifying postural alignment in sitting has been developed that uses a video-based system [8]. The aim of the study reported here was to explore the potential for an automated method to establish use of the upper limb component of the CKC. This was achieved by: i) defining the clinical rules to assess the upper limb kinetic chain status through video recordings; ii) formulating a method to replicate the clinical rules with quantities that could be measured and classified objectively; and iii) testing the extent to which the objective method replicates the clinical judgement. Initial development was performed with a group of healthy adults to eliminate the complications associated with compromised motor control. The system was then tested in a real clinical context with a group of children with $\mathrm{CP}$.

\section{Methods}

\subsection{Ethics}

This study was a preliminary technical component to a wider investigation. Ethical approval for the complete study was obtained from the NHS Health Research Authority (NRES Committee South Central, United Kingdom) and from the Manchester Metropolitan University (MMU) Ethics Committee. The study was conducted in accordance with the Declaration of Helsinki guidelines.

\subsection{Participants}

Two groups of participants were recruited: an adult group (Adult-group) of 3 males, 2 females, mean age $28 \pm 4$ years, mean height $1.72 \mathrm{~m} \pm 0.09$, and weight $73.1 \mathrm{~kg} \pm 10.2$ tested at MMU; and a child group (Child-group) of 4 males, 1 female, mean age 8.4 \pm 4.62 years, mean height $1.1 \mathrm{~m} \pm 0.27$ and weight $24.16 \mathrm{~kg} \pm 10.8$ tested at The Movement Centre (TMC, Oswestry, Shropshire, United Kingdom). All adults were 
healthy with a body mass index $<29 \mathrm{~kg} \cdot \mathrm{m}^{-2}$. All children had a diagnosis of cerebral palsy and were participating in Targeted Training (TT) therapy at TMC. All adults gave written informed consent for their participation. Children's parents provided written informed consent with child assent where possible. To allow accurate palpation of anatomical landmarks for marker placement, adults wore a tight pair of shorts with men leaving their upper body free of clothing and women wearing a tight vest. Children wore only their underwear, nappy or shorts as usual for their clinical assessments.

\subsection{Procedures}

All participants sat in an upright aligned posture on a bench free of back or arm support. The height of the bench was adjusted to ensure each participant's feet were flat on the floor with knees and hips flexed at $90^{\circ}$. Adults performed a sequence of twelve arm movements that represented both no-support, such as both arms in the air to the sides or the front, and support/contact such as hands on the bench, legs or head. Six trials were recorded per participant with different segmental levels of trunk control tested (Upper-Thoracic, UT; Mid-Thoracic, MT; Lower-Thoracic, LT; UpperLumbar, UL; Lower-Lumbar, LL; and free sitting, FS) following the SATCo guidelines [3]. The trunk was supported manually directly beneath the tested segment resulting in 'unsupported segments' above the manual support: arms (tip of the fingers to axillae), head and unsupported segments of the trunk.

Children were recorded during the routine SATCo performed as part of their TT therapy.

\subsection{Apparatus and measurements}

Data were collected simultaneously using a 3D motion capture system and one video camera.

\subsubsection{D Motion Capture}

Motion data was collected using a ten-camera system (Vicon Nexus, Oxford Metrics, Oxford, UK) at a frequency of $100 \mathrm{~Hz}$. Reflective markers were used to define the Head, Trunk and Pelvis segments, and to track the position of the right and left Elbow 
and Hand (Figure 1). Hands and Elbows were selected as representative upper limb landmarks.

Marker reconstruction and gap filling used Vicon-Nexus software (version 1.8.5). Processing was performed using Visual 3D (v.5.01, C-motion, Germantown, MD, USA); marker trajectories were lowpass filtered at $6 \mathrm{~Hz}$. Data was exported to Matlab (Mathworks, Cambridge, MA) for further analysis.

\subsubsection{Video recording}

Video was recorded at $25 \mathrm{~Hz}$ from one video camera (JVC, HD Everio RX110) mounted on a levelled tripod placed directly in front of the Adult-group at a constant distance of $3.90 \mathrm{~m}$ and constant height of $0.90 \mathrm{~m}$. For the Child-group the camera was placed at right diagonal front (approximately $45^{\circ}$ ) to allow the parent to stand in front of the child without obstructing the camera view. The camera was at a constant distance of $2.5 \mathrm{~m}$ and a constant height of $0.75 \mathrm{~m}$. Either front or oblique views are permissible for SATCo.

A second lateral view camera was used to confirm those trials where the head-trunk was vertically aligned and only those trials were processed.

\subsection{Data processing and analysis}

The Vicon and video were synchronised prior to analysis using an initial manual synchronisation followed by automated fine tuning using cross correlation.

\subsubsection{Clinical identification of Open-CKC}

The clinical classification of CKC status was performed by five clinicians familiar with this process (5-20 years of daily use). Assessors followed a defined clinical rule to assess the upper limb kinetic chain status from video recordings. This rule was: a Controlled-Kinetic-Chain is open when there is no contact between an unsupported segment and any other part of the body or any external objects. 'Contact' includes firm or light touch; 'external objects' include the supporting bench, toys, parent's hands and the hands supporting the trunk. Definition and assessment of the aligned posture in sitting has been described elsewhere [3, 8]. 
Open-CKC frames were identified from both the adult and child videos and frame numbers exported to Matlab for further analysis. The collective classification of all assessors was calculated by the mode classification for each frame.

Inter-assessor reliability was tested using a two-way mixed, absolute, average measures intraclass correlation coefficient (ICC 3,1) for each group. Intra-assessor reliability was tested for one of the assessors with 49 randomly selected videos from both groups.

\subsubsection{Automated identification of Open-CKC.}

For the automated (Vicon) classification of Open-CKC the classification rule was simplified to the location of four markers (both hands, elbows) in relation to the body and supporting bench. The body was represented by a 3D cylindrical volume covering the head-trunk and pelvis, and the bench was defined as the volume below the trochanteric markers (Figure 1). These two volumes were termed 'supported-volume'. The shortest distance from the hands and elbows to the supported-volume was calculated by customised Matlab code (Figure 2-A, B). An Open-CKC was present when all distances (both hands and elbows) were $>t \mathrm{~mm}$, where the threshold $(t)$ was an adjustable parameter (Figure 1,Figure 2-C, D). Three methods for setting $t$-values were used: i) $t=0$ (unfitted); ii) adjusting $t$ using an optimisation routine to maximise agreement with the collective clinical assessment (fitted); and iii) using generalised fixed values not requiring assessor judgement (fixed-values).

\subsubsection{Agreement between clinical and automated methods}

The agreement between the automated and the collective clinical classification of Open-CKC was calculated as the percentage of time during which the classifications were the same for each trial (Figure 2-E, F). For comparison, the mean percentage agreement between individual assessor and the collective clinical classification was also calculated.

Statistical difference between processing agreement methods was calculated with a repeated measures ANOVA for each group. The differences between segmental levels for each group was assessed using a univariate analysis for each processing method. 


\section{Results}

Twenty-nine Adult-group trials and 52 Child-group trials were analysed separately.

The clinical inter-assessor consistency of Open-CKC identification was excellent for both groups (Adult-group ICC $=0.96$, Child-group ICC $=0.95$ ). Intra-assessor reliability was also excellent $(\mathrm{ICC}=0.89)$.

The unfitted, fitted and fixed-values clinical $v$ automated agreements calculation were significantly different between methods $(68.26 \% \pm 15.7,88.32 \% \pm 5.3$ and $80.80 \% \pm 3.1$ mean $\pm S D$ respectively for unfitted, fitted and fixed-values) for the Adult-group $\left(F_{1,23}=260.36 p<0.001\right)$ and for the Child-group $(48.3 \% \pm 33.9,89.84 \% \pm 10.274 .31 \%$ \pm 21.5 as previous) $\left(F_{1.32,92}=41.07, p<0.001\right)\left(F_{1.32,92=41.07, p<0.001)}\right.$ (Figure $\left.3-A\right)$.

The clinical $v$ automated agreements (unfitted) were significantly different $(p \leq 0.001)$ between the UT and all the other segmental levels in the Adult-group, and between the UT and MT, LL and FS (both $p<0.05$ ) for the fitted processing (Figure 3-A). There were no differences for the fixed-values processing. In the Child-group there was no significant difference between segmental levels for any of the agreement methods (Figure 3-A).

For the fitted agreement the optimal $t$-values are presented in Figure 3-B. The Adultgroup shows larger $t$-values for the UT $(190.8 \mathrm{~mm})$ and MT $(186.6 \mathrm{~mm})$ segmental levels while the Child-group shows larger $t$-values for the UT $(113.7 \mathrm{~mm})$ and LT $(83.8 \mathrm{~mm})$ segmental levels. This information was used to define the threshold values for the fixed-values agreement at $200 \mathrm{~mm}$ for UT and MT segments, $100 \mathrm{~mm}$ for other segments in Adult-group and $150 \mathrm{~mm}$ for the UT segment and $50 \mathrm{~mm}$ for all other segments in the Child-group. 


\section{Discussion}

The full classification of a Controlled-Kinetic-Chain (CKC) requires knowledge of both head-trunk alignment and the position of the upper limbs. This study investigated the methods required to translate the clinical classification of the upper limb component of a CKC into an automated objective method suitable for application in a physical therapy practice for example with children who have CP.

An objective automated system should incorporate the subjective rules that are already embodied within the existing clinical practice. It should also be 'clinicallyfriendly' and not disrupt the normal practice routine, it should be 'child-friendly' (i.e. preferably without adhesive markers) and able to collect clean data within a crowded (visual) environment. Finally, an objective system should be simple for clinicians to use. This study has taken the first steps towards a clinically-friendly objective automated measure by: i) making explicit and then testing a precise formulation of the clinical rules; and ii) exploring whether a reduced, minimum set of rules could objectively replicate the clinical classification.

Results showed that the clinician intra- and inter-assessor reliability was excellent with either a frontal view (Adult-group) or an oblique view (Child-group). Humans can extract 3D information from a single camera view and extracting this full 3D information automatically will be technically challenging. Thus, the next step taken in this study was to determine the minimum $3 \mathrm{D}$ information that might be required by an automated system.

This study describes two groups of participants. These groups differ so widely that it was inappropriate to consider the Adult-group a 'control-group'; the Adult-group, however, served in the initial development to eliminate the complications associated with compromised motor control.

Results for the unfitted method showed that it was possible to classify Open-CKC v Closed-CKC using only the positions of the participant's hands and elbows in relation to the supported-volume. However, the relatively low percentages of agreement between clinicians and this simple method, particularly at higher levels of support, were a clear indication that this method was not capturing sufficiently what clinicians observe from video. 
Results for the fitted method showed that the agreement with the clinical judgement improved substantially by adding a single adjustable parameter. This parameter $(t)$ increased the thickness of the supported-volume, incorporating the supporting hands and ensuring clearance with the participant's body. The $t$-value was adjusted to maximise agreement with the clinical assessment. Furthermore, a larger $t$-value, particularly at higher levels of support (UT and MT), matched better with the clinical assessment. This implies that during a SATCo to test UT segmental level, the clinician's hands providing trunk support also potentially provide external mechanical support to the upper limbs. An Open-CKC is only demonstrated when the upper limbs are clear by a margin of error represented by values required for $t$.

Applying parameter $t$ without using clinical assessment was tested in the fixed-values method. Results showed that it was possible (more than $70 \%$ agreement), to replicate the clinical judgement using fixed values of $t$ that were participant invariant and level of segmental support specific. Using general values in this way implies that the method is fully automated i.e. clinical judgment is not needed to modify the $t$. However, this study used relatively small groups of participants; increasing the number of participants could help to refine the general $t$-values and increase the fixed-values reliability. Furthermore, it remains possible that this automated rule could be improved further using participant specific measurements.

The work developed in the present study used a 3D motion capture system to support the concept. There are, however, several difficulties with this system. A clinician can detail the volume of the upper arm and see its relation to the supporting hands or the participant's body and can distinguish the presence of light touch that results in a Closed-CKC. A clinician can also easily identify external supporting elements from video such as a child's contact with parents' hands. In contrast, the 3D system was based on a simplified model of the upper arms and, even if this model was more complex, it would still be difficult for a 3D motion capture system to identify light touch. Furthermore, external objects can only be recognised by a 3D system if they have reflective markers.

This assessment overall (alignment and CKC status) will measure the head/trunk control demonstrated by a child. It is known that typically developing infants achieve independent sitting between 6 and 8 months of age [9]. The full assessment of 
alignment and CKC status will allow measurement of this process in typical development and of the emergence of trunk control in children with CP; this may lead to greater understanding of control elements related to immaturity and/or to dysfunction. The children in this study could be showing trunk control that is primarily related to their dysfunction but this cannot be stated definitively at this stage of development of the quantitative and automated tool. Although the position of the hands and arms in relation to independent sitting has been studied before both using video analysis [10] and a 3D motion capture system [11], the analysis was related to symmetrical or asymmetrical reaching and to the qualities of reaching and manipulation. As far as could be determined from the literature, the use of the upper limbs to compensate for poor trunk control in sitting has not been previously studied.

This study has demonstrated that the upper limb component of a CKC can be identified objectively and that it matches with the clinical judgement. The shortcomings of a 3D system have also been identified. These difficulties can be overcome by the development of a video-based system using the factors established in this study to complement clinical assessments in neurodisability such as cerebral palsy.

\section{Conclusion}

This study addressed the classification of Open-CKC required for the clinical assessment of trunk control status in children with cerebral palsy. Results demonstrated that, if a participant is sitting with an aligned head-trunk, a frontal or oblique camera provides sufficient information for clinicians to make a reliable, objectively supported, clinical analysis of upper limb Open-CKC in children with cerebral palsy. The automated objective method reduced the clinical judgement to measurement of the position of the participant's hands and elbows in relation to a defined supported-volume of the head-trunk using a 3D motion capture system (Vicon). While this simplified objective measure was less robust than the clinical judgment it demonstrates the main rules required to analyse Controlled-Kinetic-Chain status and thus justifies future investment in application of advanced image analysis techniques to enable automatic CKC classification in a clinically-friendly method. 
Conflict of interest

None 


\section{References}

[1] Jensen J, van Zandwijk R. Biomechanical aspects of the development of postural control. In: Korff T, De Ste Croix M, editors. Paediatric biomechanics and motor control: theory and application. Abingdon, Oxon: Routledge research in sport and exercise science; 2012. p. 139-59.

[2] Butler P, Major R. The missing link? Therapy issues of open and closed chains. Physiotherapy. 2003;89:465-70.

[3] Butler P, Saavedra SL, Sofranc M, Jarvis S, Woollacott MH. Refinement, reliability, and validity of the Segmental Assessment of Trunk Control. Pediatr Phys Ther. 2010;22:246-57.

[4] Pountney TE, Cheek L, Green E, Mulcahy C, Nelham R. Content and Criterion Validation of the Chailey Levels of Ability. Physiotherapy. 1999;85:410-6.

[5] Russell DJ, Rosenbaum PL, Avery LM, Lane M. Gross motor function measure (GMFM-66 and GMFM-88) user's manual. First ed. London: Mac Keith Press; 2002.

[6] Heyrman L, Molenaers G, Desloovere K, Verheyden G, De Cat J, Monbaliu $\mathrm{E}$, et al. A clinical tool to measure trunk control in children with cerebral palsy: the Trunk Control Measurement Scale. Res Dev Disabil. 2011;32:2624-35.

[7] Reid DT. Sitting Assessment for Children with Neuromotor Dysfunction SACND. San Antonio, Texas: Therapy Skill Builders; 1997.

[8] Sánchez MB, Loram I, Darby J, Holmes P, Butler PB. A video based method to quantify posture of the head and trunk in sitting. Gait Posture. 2017;51:1817.

[9] Saavedra SL, van Donkelaar P, Woollacott MH. Learning about gravity: segmental assessment of upright control as infants develop independent sitting. J Neurophysiol. 2012;108:2215-29.

[10] Rochat P. Self-Sitting and Reaching in 5- to 8-Month-Old Infants: The Impact of Posture and Its Development on Early Eye-Hand Coordination. J Mot Behav. 1992;24:210-20.

[11] Nogueira SF, Figueiredo EM, Gonçalves RV, Mancini MC. Relation between hand function and gross motor function in full term infants aged 4 to 8 months. Braz J Phys Ther. 2015;19:52-60. 


$$
\text { OpenCKC }=\text { all }\left(\left[\begin{array}{l}
d 1 \\
d 2 \\
d 3 \\
d 4
\end{array}\right]>t\right)
$$

where $t$ is an adjustable threshold

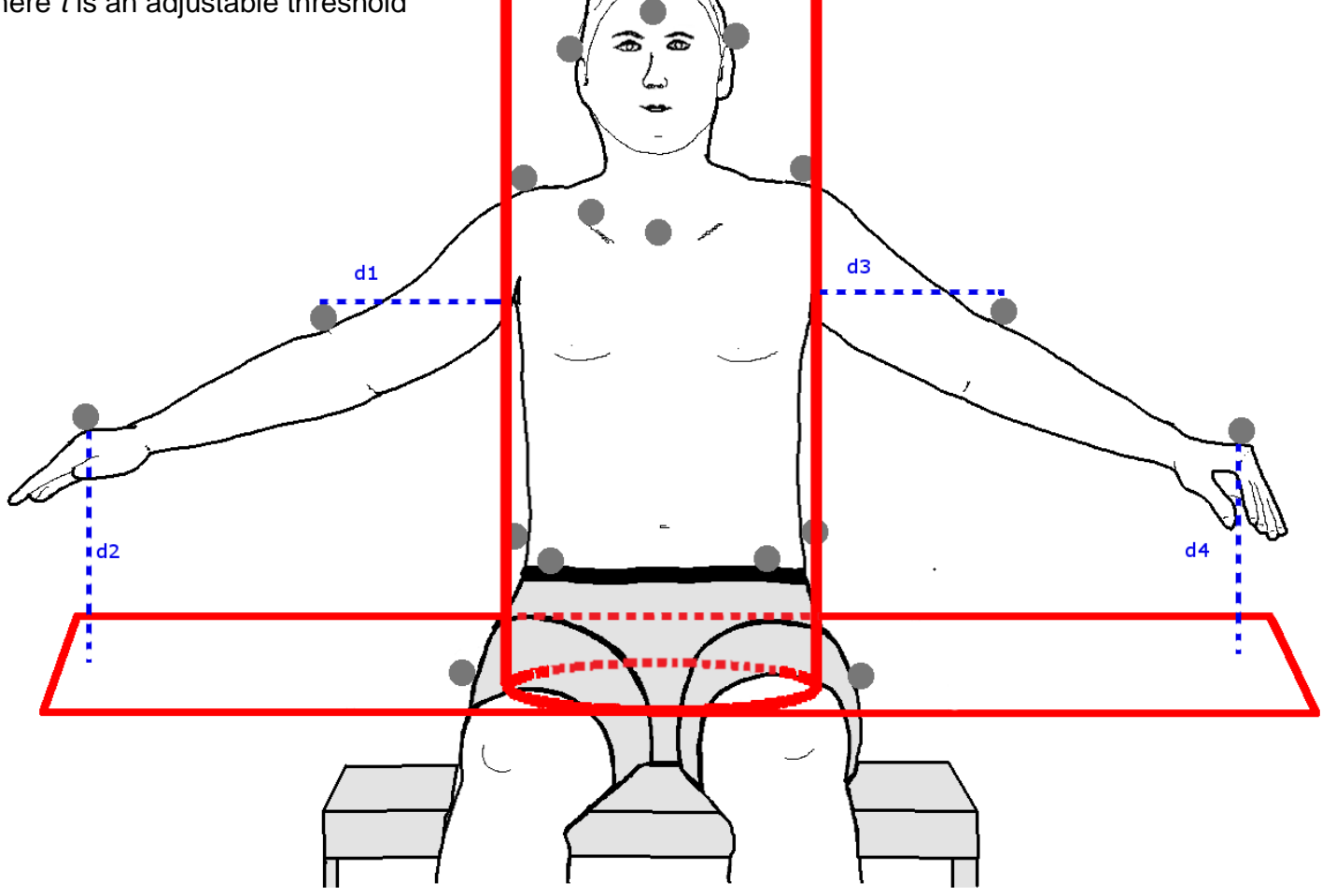

Figure 1 Marker locations and supported-volume

Dots show Vicon marker locations: forehead, middle of the right clavicle, left and right acromion process of the scapula, lateral condyle of the humerus (elbow), head of the third metacarpal bone, lliac crest, anterior superior iliac spine and greater trochanter. The red cylinder and plane represent the volume that defined a Closed-CKC. Dashed blue lines show the shortest distances $\left(d_{1-4}\right)$ from each of the hands and elbows to the supported-volume surface for this given posture. 
A)

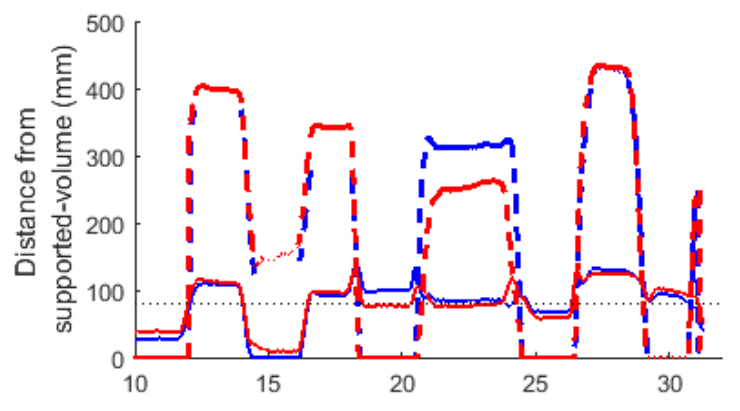

C)

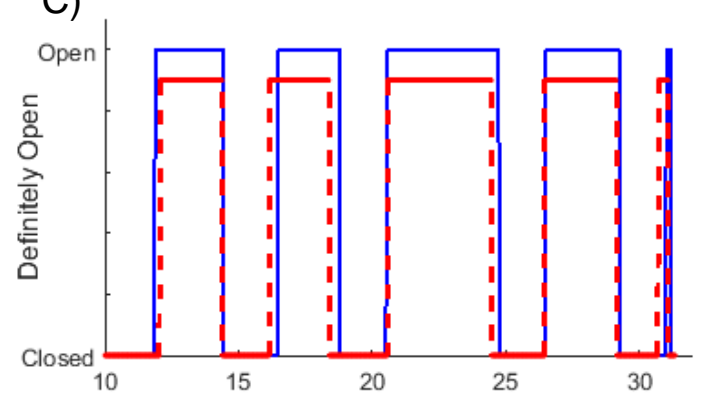

E)

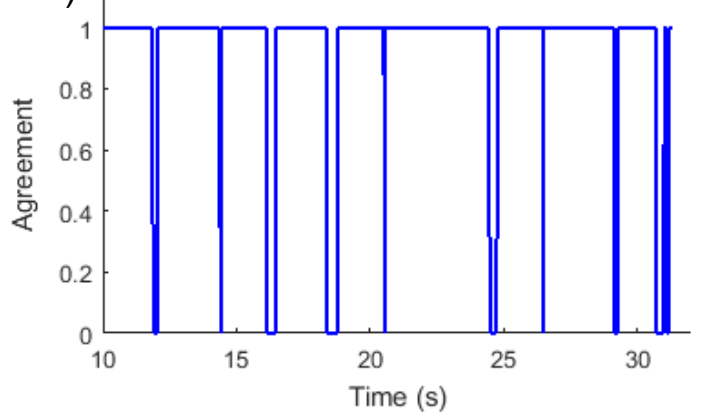

B)

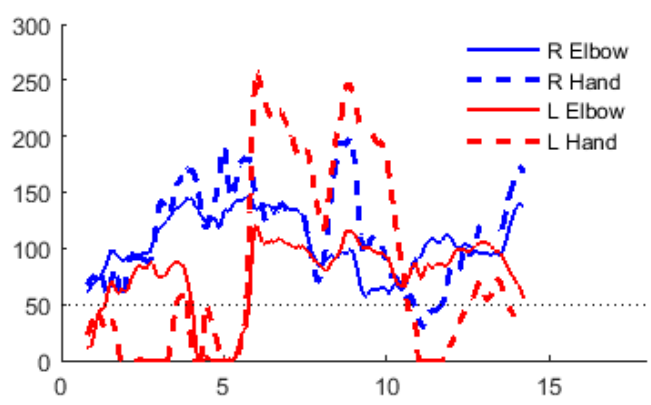

D)

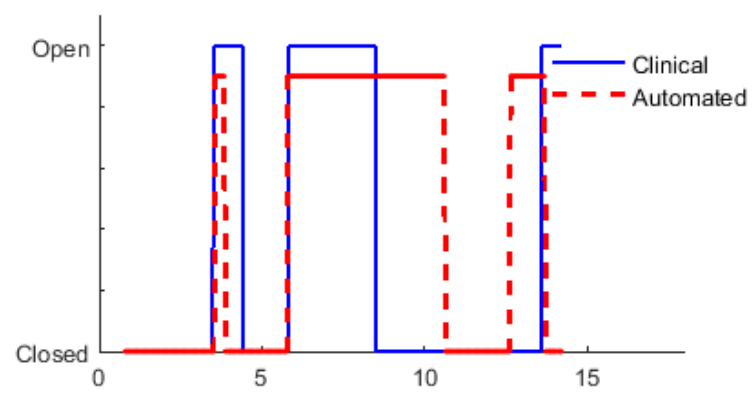

F)

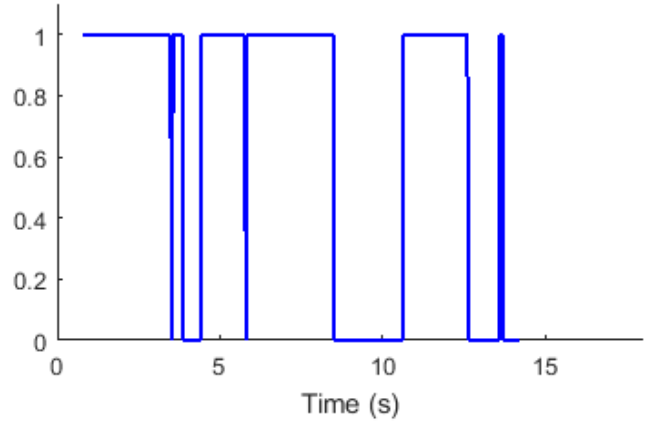

Figure 2 Representative examples of the automated tracking of the upper limb, classification of Open CKC and calculated agreement over time.

Showing a representative trial example for the Adult-group (panels $A, C, E$ ) and Child-group (panels $B, D, F)$. A,B) Automated tracking of the upper limb (left, red line; right, blue line) shows the position of the hands (dash) and elbows (continuous) relative to the supported-volume. The black dotted line shows the t-values used for calculations. C,D) Classification of the Open-CKC for the clinical (blue line) and the automated (dash red line, reduced height for visibility) assessment using the fitted method for the adult and the fixed-values method for the child. E,F) Shows the agreement between clinical and automated classification $(92.4 \%$ for the adult. $68.5 \%$ for the child). 


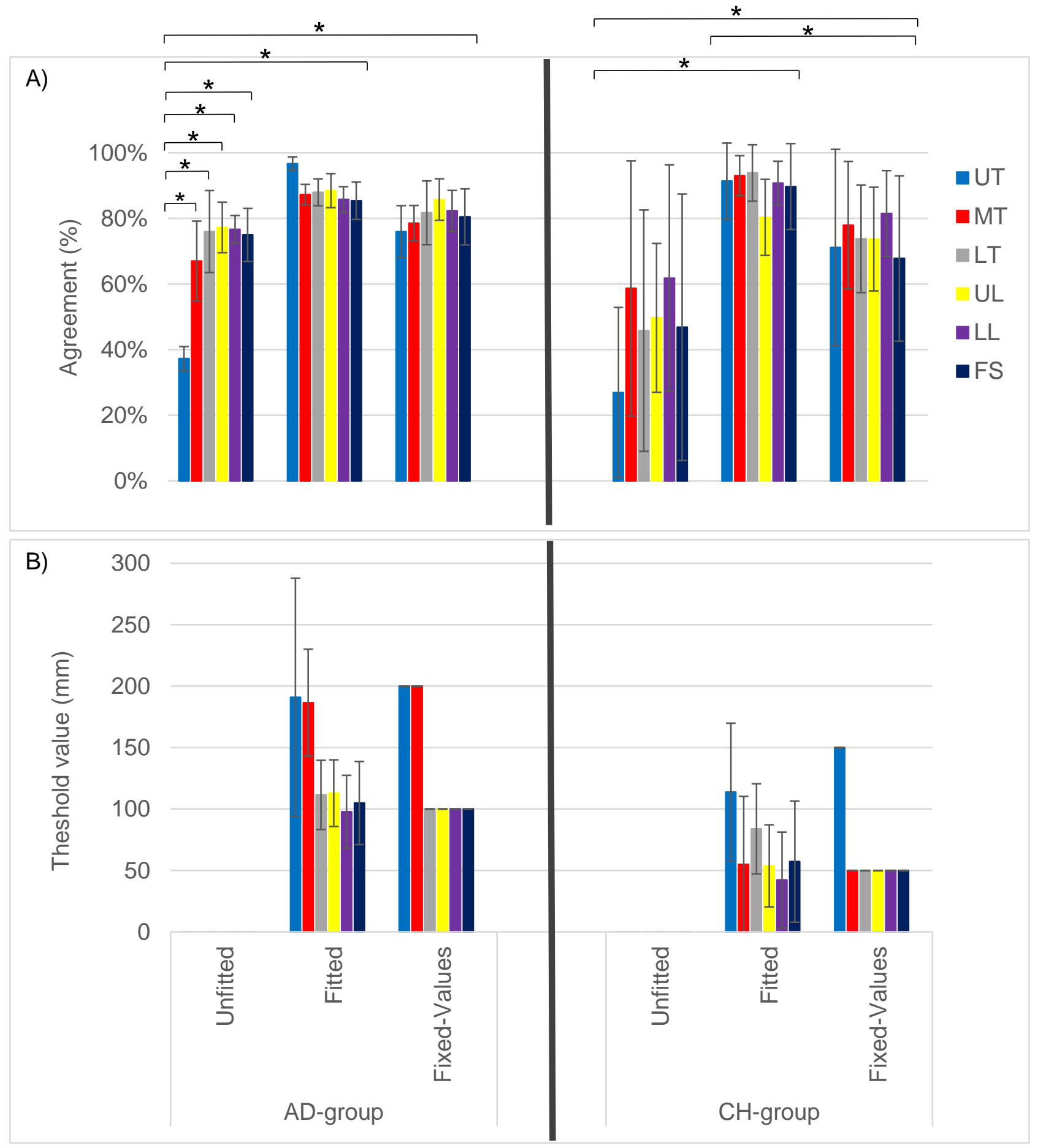

Figure 3 Calculated agreement between the human and the objective identification of Open-CKC.

A) Showing the mean collective percentage of agreement for the Adult-group (AD-group) and the Child-group ( $\mathrm{CH}$-group) for all processing methods (unfitted, fitted and fixed) and the standard deviation (error bars). Agreement is presented separately for each segment tested (Upper-Thoracic, UT; Mid-Thoracic, MT; LowerThoracic, LT; Upper-Lumbar, UL; Lower-Lumbar, LL; and Free Sitting, FS). +indicates significant difference, $p<0.05$. * indicates strong significant difference, $p<0.001$. B) Showing threshold values for the fitted and the fixed agreement calculations of the various segments. 